\title{
Evaluation of antioxidant status and Serum lipoprotein (a) in coronary heart diseases
}

\author{
Sudeep. R. Lokapure ${ }^{1}$, Jano Zore ${ }^{1}$, Kavitha. Y. Ullikashi ${ }^{2}$, Chitra Dhume ${ }^{1}$ \\ ${ }^{1}$ Department of Biochemistry,Goa Medical college,Bambolim-Goa \\ ${ }^{2}$ Subject Matter Specialist (Home science), Krishi vigyan kendra,Hagari,Bellary,Karnataka
}

\section{Email address:}

drsudeeplokapure@gmail.com (S. R. Lokapure)

\section{To cite this article:}

Sudeep. R. Lokapure, Jano Zore, Kavitha. Y. Ullikashi, Chitra Dhume. Evaluation of Antioxidant Status and Serum Lipoprotein(a) in Coronary Heart Diseases. Advances in Biochemistry. Vol. 2, No. 1, 2014, pp. 1-8. doi: 10.11648/j.ab.20140201.11

\begin{abstract}
Coronary heart disease (CHD) has become the most important cause of premature morbidity and mortality. The chief risk factors for CHD includes Smoking, Hypertension, Diabetes mellitus, Hypercholesterolemia, Low high density lipoprotein, Obesity, Mental stress, Type A personality and Genetic factors. Lipoprotein (a) has been established as a strong independent risk factor for premature $\mathrm{CHD}$, which is highly thrombogenic and antifibrinolytic which begins to block the arteries much earlier than other risk factors. This study is planned to determine the antioxidant status and to estimate Serum lipoprotein (a) levels in patients of CHD and in patients with risk factors for CHD. Among all the patients, majority of patients belongs to upper and middle class. In routine investigations an increase in serum cholesterol, serum LDL, TC/HDL and LDL/HDL ratio were seen in all five groups including MI, HT, DM, Smokers and obesity cases, which were statistically significant. Serum Triglycerides were increased in MI, DM, Smokers and obesity cases, which were statistically significant, where as significant increase was not seen in HT cases. Serum VLDL showed a statistically significant increase in MI, Smokers and obesity cases, where as significant increases was not seen in HT and DM cases. Blood glucose showed a significant increase in DM cases, where as no significant increase were seen in MI, HT, smokers and obesity cases. A statistically significant decrease in serum HDL were seen in all five groups including MI, HT, DM, Smokers and obesity cases. In special investigations, there is significant increase in MDA, Lipoprotein (a) levels and significant decrease of antioxidants such as blood glutathione, Serum vitamin E, vitamin C and vitamin A in cases of coronary heart diseases and also in cases with risk factors for Coronary heart diseases including hypertension, diabetes mellitus, smoking and obesity. Hence, it is therefore concluded that MDA, Lipoprotein (a) and antioxidants like glutathione, vitamin E, Vitamin C and Vitamin A, be evaluated as biochemical parameters for preclinical assessment of "at risk group" for Coronary heart diseases and for assessing and monitoring cases of Coronary heart diseases.
\end{abstract}

Keywords: Coronary Heart Diseases, Lipoprotein (A), MDA, Antioxidants

\section{Introduction}

Coronary heart disease (CHD) has become the most important cause of premature morbidity and mortality. With the advancement of medical science in the $20^{\text {th }}$ century, there has been a remarkable increase in the life expectancy throughout the world by controlling infections and consequently more number of deaths are recorded due to degenerative causes like coronary atherosclerosis. CHD is very important medical problem and in spite of a large number of researches for diagnosis and treatment, it takes a huge toll of human lives all over the globe. CHD produces localized ischaemia of the myocardium and when the occlusion is complete, myocardial infarction takes place.
There are various risk factors, presence of which makes person more prone to develop CHD, the risk factors for CHD includes smoking, hypertension, diabetes mellitus, obesity and others. Lipid peroxidation is a free radical mediated reaction, it is not possible to measure free radicals, so lipid peroxidation is measured in terms of Malondialdehyde (MDA), which is end product of lipid peroxidation. There are natural protective molecule which scavenge free radicals called antioxidants e.g., Glutathione, Vitamin E, Vitamin C and vitamin A. The level of these antioxidants will determine the antioxidant status. Lipoprotein (a) $[\mathrm{Lp}(\mathrm{a})]$ is a specific class of lipoprotein particles consists of apo (a) which is linked with apo $B_{100}$ by a disulfide bond. $\mathrm{Lp}$ (a) now been established as a strong 
independent risk factor for premature CHD, which is highly thrombogenic and antifibrinolytic, which begins to block the arteries much earlier than other risk factors.

To know the co-relation of total antioxidant status by the parameters mentioned above and concentration of $\mathrm{Lp}(\mathrm{a})$ and to evaluate of whether they can be used as monitoring indices in Coronary heart diseases, The present study is planned with following objectives.

1 To study the antioxidant status (mainly antioxidants- vitamin $\mathrm{E}$, vitamin $\mathrm{C}$ and vitamin $\mathrm{A}$ ) in patients of Coronary heart diseases and in patients with risk factors including smoking, Hypertension, Diabetes mellitus and Obesity, against normal healthy controls to evaluate a critical level if possible.

2 Estimation of Lipoprotein (a), a independent risk factor for Coronary heart diseases.

3 Estimation of malondialdehyde (MDA), which indicates the extent of Lipid peroxidation in Coronary heart disease.

4 Estimation of Glutathione (GSH), a protection against peroxidation.

5 To evaluate whether there can be used as monitoring indices in Coronary heart diseases.

\section{Materials and Methods}

\subsection{Selection of Group}

The present study comprises of 80 patients, 20 patients of myocardial infarction, 60 patients with risk factor of Coronary heart disease (CHD), including Hypertension, Diabetes mellitus, Smoking and Obesity, seeking medical care in Goa Medical college Hospital, Bambolim during the period of November 2004 to October 2005. 20 healthy age and sex matched subjects served as controls. All the patients in the study group and control group were aged between 30-60 years. In both the groups a detail history was obtained and a thorough clinical examination was carried out.

\subsection{Collection of Blood Samples}

About $5 \mathrm{ml}$ of blood samples were collected in plain bulb and were allowed to clot. After one hour the serum was separated by centrifuging at $2500 \mathrm{rpm}$ for 5 minutes at room temperature. Serum was used for measurement of MDA, Vitamin E and Lp(a). About $5 \mathrm{ml}$ of blood samples were collected in flouride bulb, plasma was separated and used for measurement of Vitamin C, Vitamin A and whole blood for measurement of blood glutathione.

All estimation were done within 24-48 hours after sample collection.

\subsection{Special Investigation}

Special investigation like serum Lp(a) estimated using Turbilatex quantitative turbidimetric test, serum MDA estimated with principle of precipation of lipoprotein by adding 20\% TCA and treating with TBA (Thiobarbituric acid) in sodium sulphate to form Chromogen, which forms complex in boiling water and extracted in butanol, measured at $530 \mathrm{~nm}$ (SATOH, 1978). Blood glutathione estimated by photometric method adopted by Beutler (1963) using 5-5, Di-thiobis 2-Nitro benzoic acid (D.T.N.B). Serum vitamin E determined as a serum tocopherol by their reduction of ferric to ferrous ion which then form a red complex with alpha dipyridyl (Baker and Frank, 1968). Vitamin C determined as plasma ascorbate by using 2,6dichlorophenolindophenol titration method. Vitamin-A determined as a retinol and carotenes in serum using the carr price reaction (Kimble 1938-39, Kaser and Stekol 1943).

\subsection{Routine Investigation}

Blood glucose determined by Folin-wu's method, total serum cholesterol estimated using Liebermann Burchard reaction (Kim and Goldberg's method), Serum triglycerides estimated by GPO-PAP method (Enzymatic colorimetric method), serum HDL cholesterol by phospo tungstate magnesium method, LDL (Low density lipoprotein cholesterol) and VLDL (very low density lipoprotein cholesterol) is calculated by Frieldwald formula.

\section{Results and Discussion}

The present study comprises of 100 subjects, which includes 80 cases of Coronary heart diseases of which 20 cases of Myocardial Infarction (MI), 60 cases with risk factors for CHD including Hypertension (HT), Diabetes mellitus (MI), Smoking and Obesity (Table 1) and 20 age matched controls.

Table 1. Distribution of total study subjects

\begin{tabular}{lc}
\hline Group & Number of cases \\
\hline Myocardial Infarction & 20 \\
Hypertension & 15 \\
Diabetes Mellitus & 15 \\
Smokers & 15 \\
Obesity & 15 \\
Control & 20 \\
Total & $\mathbf{1 0 0}$ \\
\hline
\end{tabular}

Table 2. Age incidence of the patients in study group and control

\begin{tabular}{ccccc}
\hline $\begin{array}{c}\text { Age in } \\
\text { years }\end{array}$ & $\begin{array}{c}\text { Myocardial } \\
\text { Infarction (20) }\end{array}$ & $\begin{array}{c}\text { Percentag } \\
\text { e \% }\end{array}$ & $\begin{array}{c}\text { Hypertens } \\
\text { ion (15) }\end{array}$ & $\begin{array}{c}\text { Percentag } \\
\text { e \% }\end{array}$ \\
\hline 30 to 40 & 2 & 10 & 1 & 6.6 \\
41 to 50 & 7 & 35 & 1 & 6.6 \\
51 to 60 & 11 & 55 & 13 & 86.6 \\
\hline
\end{tabular}


continue

\begin{tabular}{ccccc}
\hline $\begin{array}{c}\text { Age in } \\
\text { years }\end{array}$ & $\begin{array}{c}\text { Diabetes } \\
\text { Mellitus (15) }\end{array}$ & $\begin{array}{c}\text { Percentag } \\
\mathbf{e} \%\end{array}$ & $\begin{array}{c}\text { Smokers } \\
\mathbf{( 1 5 )}\end{array}$ & $\begin{array}{c}\text { Percentag } \\
\mathbf{e} \%\end{array}$ \\
\hline 30 to 40 & 1 & 6.6 & 1 & 6.6 \\
41 to 50 & 2 & 13.3 & 3 & 20 \\
51 to 60 & 12 & 80 & 11 & 73.3 \\
\hline & & continue & & \\
\hline $\begin{array}{c}\text { Age in } \\
\text { years }\end{array}$ & $\begin{array}{c}\text { Obesity } \\
\text { (15) }\end{array}$ & $\begin{array}{c}\text { Percentage } \\
\text { \% }\end{array}$ & $\begin{array}{c}\text { Control } \\
\mathbf{( 1 5 )}\end{array}$ & $\begin{array}{c}\text { Percentage } \\
\text { \% }\end{array}$ \\
\hline 30 to 40 & 2 & 13.3 & 5 & 25 \\
41 to 50 & 2 & 13.3 & 6 & 30 \\
51 to 60 & 11 & 73.3 & 9 & 45 \\
\hline
\end{tabular}

Table 3. Distribution of cases according to their Socio-economic status

\begin{tabular}{|c|c|c|c|}
\hline Group & $\begin{array}{c}\text { Socio-economic } \\
\text { status }\end{array}$ & $\begin{array}{c}\text { Number of } \\
\text { pts }\end{array}$ & Percentage \\
\hline \multirow{4}{*}{$\begin{array}{c}\text { Myocardial } \\
\text { Infarction (20) }\end{array}$} & Lower & 4 & 20 \\
\hline & Middle & 6 & 30 \\
\hline & Upper & 10 & 50 \\
\hline & Lower & 3 & 20 \\
\hline \multirow[t]{2}{*}{ Hypertension (15) } & Middle & 5 & 33.3 \\
\hline & Upper & 7 & 46.6 \\
\hline \multirow{3}{*}{$\begin{array}{l}\text { Diabetes Mellitus } \\
\text { (15) }\end{array}$} & Lower & 2 & 13.3 \\
\hline & Middle & 5 & 33.3 \\
\hline & Upper & 8 & 53.3 \\
\hline \multirow{3}{*}{ Smokers (15) } & Lower & 5 & 33.3 \\
\hline & Middle & 4 & 26.6 \\
\hline & Upper & 6 & 40 \\
\hline \multirow{3}{*}{ Obesity (15) } & Lower & 2 & 13.3 \\
\hline & Middle & 4 & 26.6 \\
\hline & Upper & 9 & 60 \\
\hline \multirow{3}{*}{ Control (20) } & Lower & 5 & 25 \\
\hline & Middle & 7 & 35 \\
\hline & Upper & 8 & 40 \\
\hline
\end{tabular}

Table 4. Showing the chief symptoms in 20 patients of myocardial infarction

\begin{tabular}{ccc}
\hline Symptoms & No. of patients & Percentage \% \\
\hline Chest pain & 18 & 90 \\
Sweating & 16 & 80 \\
Vomiting & 9 & 45 \\
Breathlessness & 6 & 30 \\
Palpitation & 8 & 40 \\
Giddiness & 4 & 20 \\
\hline
\end{tabular}

Table 5. Table Showing the levels of serum Lipoprotein (a) in cases and controls.

\begin{tabular}{lccc}
\hline Group & $\begin{array}{c}\text { Range of serum } \\
\text { Lipoprotein (a) } \\
\text { (mg/d) }\end{array}$ & $\begin{array}{c}\text { Mean } \pm \text { S.D. } \\
\text { (mg/dl) }\end{array}$ & $\begin{array}{c}\text { P } \\
\text { value }\end{array}$ \\
\hline $\begin{array}{l}\text { Myocardial Infarction } \\
(20)\end{array}$ & $28-79$ & $43.59 \pm 10.22$ & $<0.001$ \\
$\begin{array}{l}\text { Hypertension (15) } \\
\begin{array}{l}\text { Diabetes Mellitus } \\
(15)\end{array}\end{array}$ & $25-44$ & $33.83 \pm 6.29$ & $<0.001$ \\
$\begin{array}{l}\text { Smokers (15) } \\
\text { Obesity (15) }\end{array}$ & $29-45$ & $36.57 \pm 5.29$ & $<0.001$ \\
Control (20) & $22-38$ & $33.93 \pm 3.57$ & $<0.001$ \\
(Reference group) & $13-18$ & $29.07 \pm 3.42$ & $<0.001$ \\
\hline
\end{tabular}

ANOVA F: $50.155 \quad \mathrm{~F}:(5,94) \mathrm{P}<0.001$

As the $\mathrm{P}$ value is $\mathrm{P}<0.001$, the result is highly significant.

Table 6. Table Showing the levels of MDA in cases and controls.

\begin{tabular}{lccc}
\hline Group & $\begin{array}{c}\text { Range of serum } \\
\text { MDA }(\boldsymbol{\mu m o l} / \mathbf{l})\end{array}$ & $\begin{array}{c}\text { Mean } \pm \text { S.D. } \\
(\boldsymbol{\mu m o l} / \mathbf{l})\end{array}$ & $\begin{array}{c}\text { P } \\
\text { value }\end{array}$ \\
\hline $\begin{array}{l}\text { Myocardial } \\
\text { Infarction (20) }\end{array}$ & $12.2-20$ & $15.98 \pm 2.06$ & $<0.001$ \\
Hypertension (15) & $9-17.3$ & $12.64 \pm 2.64$ & $<0.001$ \\
$\begin{array}{l}\text { Diabetes Mellitus } \\
\text { (15) }\end{array}$ & $10-17.7$ & $13.77 \pm 2.17$ & $<0.001$ \\
Smokers (15) & $11.5-15.5$ & $13.3 \pm 1.12$ & $<0.001$ \\
Obesity (15) & $10-13.5$ & $11.68 \pm 1.09$ & $<0.001$ \\
$\begin{array}{l}\text { Control (20) } \\
\text { Reference group) }\end{array}$ & $4.3-8.8$ & $16.76 \pm 1.20$ & \\
\hline
\end{tabular}

ANOVA F: 557.648 df : $(5,94) \mathrm{P}<0.001$

As the $\mathrm{P}$ value is $\mathrm{P}<0.001$, the result is highly significant.

Table 7. Table Showing the levels of GSH in cases and controls.

\begin{tabular}{lccc}
\hline Group & $\begin{array}{c}\text { Range of blood GSH } \\
(\mathbf{m g} \%)\end{array}$ & $\begin{array}{c}\text { Mean } \pm \text { S.D. } \\
(\mathbf{m g} \%)\end{array}$ & $\begin{array}{c}\text { P } \\
\text { value }\end{array}$ \\
\hline $\begin{array}{l}\text { Myocardial } \\
\text { Infarction (20) }\end{array}$ & $35.4-57.2$ & $48.94 \pm 5.57$ & $<0.001$ \\
$\begin{array}{l}\text { Hypertension (15) } \\
\text { Diabetes Mellitus } \\
\text { (15) }\end{array}$ & $42.5-60$ & $55.46 \pm 4.65$ & $<0.001$ \\
$\begin{array}{l}\text { Smokers (15) } \\
\text { Obesity (15) }\end{array}$ & $44.5-60$ & $52 \pm 6.61$ & $<0.001$ \\
& $52.9-62$ & $54.08 \pm 2.16$ & $<0.001$ \\
$\begin{array}{l}\text { Control (20) } \\
\text { (Reference group) }\end{array}$ & $60.1-64.3$ & $59.17 \pm 2.21$ & $>0.05$ \\
\hline
\end{tabular}

ANOVA F: 24.5338 df : $(5,94) \mathrm{P}<0.001$ 
Table 8. Table Showing the levels of alpha tocopherol in cases and controls.

\begin{tabular}{|c|c|c|c|c|}
\hline Vitamin & Group & Range of (mg/l) & Mean \pm S.D. (mg/l) & P value \\
\hline \multirow{6}{*}{$\begin{array}{l}\text { Alpha } \\
\text { Tocopherol }\end{array}$} & Myocardial Infarction (20) & $3.8-8.2$ & $5.33 \pm 1.12$ & $<0.001$ \\
\hline & Hypertension (15) & $4.1-8.3$ & $5.98 \pm 1.33$ & $<0.001$ \\
\hline & Diabetes Mellitus (15) & $4.1-10.7$ & $6.28 \pm 1.88$ & $<0.001$ \\
\hline & Smokers (15) & $4-5.4$ & $4.90 \pm 0.32$ & $<0.001$ \\
\hline & Obesity (15) & $4.5-6.6$ & $5.88 \pm 0.48$ & $<0.001$ \\
\hline & $\begin{array}{l}\text { Control (20) } \\
\text { (Reference group) }\end{array}$ & $10.7-14.4$ & $12.74 \pm 1.34$ & \\
\hline
\end{tabular}

ANOVA F: 111.959 df : $(5,94) \mathrm{P}<0.001$

As the $\mathrm{P}$ value is $<0.001$, the result is highly significant.

Table 9. Table Showing the levels of Ascorbic acid in cases and controls.

\begin{tabular}{|c|c|c|c|c|}
\hline Vitamin & Group & Range of (mg/l) & Mean \pm S.D. (mg/l) & P value \\
\hline \multirow{6}{*}{ Ascorbic acid } & Myocardial Infarction (20) & $4.4-8.0$ & $5.75 \pm 0.91$ & $<0.001$ \\
\hline & Hypertension (15) & $5-8$ & $6.76 \pm 1.05$ & $<0.001$ \\
\hline & Diabetes Mellitus (15) & $4.4-10$ & $6.72 \pm 1.69$ & $<0.001$ \\
\hline & Smokers (15) & $5-7.2$ & $6.02 \pm 0.54$ & $<0.001$ \\
\hline & Obesity (15) & $6.1-8.3$ & $7.48 \pm 0.65$ & $<0.001$ \\
\hline & $\begin{array}{l}\text { Control (20) } \\
\text { (Reference group) }\end{array}$ & $9.2-13.1$ & $11.26 \pm 1.37$ & \\
\hline
\end{tabular}

ANOVA F: 62.718 df : $(5,94) \mathrm{P}<0.001$

As the $\mathrm{P}$ value is $<0.001$, the result is highly significant.

Table 10. Table Showing the levels of Vitamin A in cases and controls.

\begin{tabular}{|c|c|c|c|c|}
\hline Vitamin & Group & Range of (mg/l) & Mean \pm S.D. $(\mathrm{mg} / \mathrm{l})$ & P value \\
\hline \multirow{6}{*}{ Vitamin A } & Myocardial Infarction (20) & $0.26-0.4$ & $0.31 \pm 0.04$ & $<0.001$ \\
\hline & Hypertension (15) & $0.28-0.43$ & $0.33 \pm 0.04$ & $<0.001$ \\
\hline & Diabetes Mellitus (15) & $0.28-0.41$ & $0.33 \pm 0.03$ & $<0.001$ \\
\hline & Smokers (15) & $0.3-0.43$ & $0.37 \pm 0.04$ & $<0.001$ \\
\hline & Obesity (15) & $0.31-0.62$ & $0.48 \pm 0.08$ & $>0.05$ \\
\hline & $\begin{array}{l}\text { Control (20) } \\
\text { (Reference group) }\end{array}$ & $0.34-0.6$ & $0.47 \pm 0.08$ & \\
\hline
\end{tabular}

ANOVA F: 25.541 df : $(5,94) \mathrm{P}<0.001$

Table 11. Table Showing the levels of serum cholesterol in cases and controls.

\begin{tabular}{clccc}
\hline Vitamin & Group & Range of (mg\%) & Mean \pm S.D. (mg\%) & P value \\
\hline & Myocardial Infarction (20) & $173-276$ & $234.0 \pm 29.12$ & $234.4 \pm 25.30$ \\
& Hypertension (15) & $180-272$ & $<0.001$ \\
Serum & Diabetes Mellitus (15) & $165-276$ & $226.9 \pm 38.03$ & $<0.001$ \\
Cholesterol & Smokers (15) & $165-290$ & $229.4 \pm 34.11$ & $26.01 \pm 29.42$ \\
& Obesity (15) & $189-289$ & $<0.001$ & $<0.001$ \\
& Control (20) & $167-192$ & \\
\hline
\end{tabular}

ANOVA F: 16.448 df : $(5,94) \mathrm{P}<0.001$

As the $\mathrm{P}$ value is $<0.001$, the result is highly significant.

Table 12. Table Showing the levels of serum Triglycerides in cases and controls.

\begin{tabular}{|c|c|c|c|c|}
\hline Vitamin & Group & Range of (mg\%) & Mean \pm S.D. $(\mathrm{mg} \%)$ & P value \\
\hline \multirow{6}{*}{ Serum Triglycerides } & Myocardial Infarction (20) & $145-251$ & $180.4 \pm 24.89$ & $<0.001$ \\
\hline & Hypertension (15) & $110-198$ & $153 \pm 23.28$ & $>0.05$ \\
\hline & Diabetes Mellitus (15) & $124-260$ & $177.6 \pm 29.88$ & $<0.001$ \\
\hline & Smokers (15) & $139-194$ & $166.6 \pm 18.85$ & $<0.001$ \\
\hline & Obesity (15) & $163-245$ & $198.0 \pm 21.61$ & $<0.001$ \\
\hline & $\begin{array}{l}\text { Control (20) } \\
\text { (Reference group) }\end{array}$ & $124-143$ & $133.1 \pm 5.65$ & \\
\hline
\end{tabular}

ANOVA F: $25.541 \mathrm{df}:(5,94) \mathrm{P}<0.001$ 
Table 13. Table Showing the levels of serum HDL in cases and controls.

\begin{tabular}{|c|c|c|c|c|}
\hline Vitamin & Group & Range of (mg\%) & $\operatorname{Mean} \pm$ S.D. $(\mathrm{mg} \%)$ & P value \\
\hline \multirow{6}{*}{ Serum HDL } & Myocardial Infarction (20) & $30-42$ & $35.75 \pm 3.25$ & $<0.001$ \\
\hline & Hypertension (15) & $36-49$ & $40.07 \pm 3.55$ & $<0.001$ \\
\hline & Diabetes Mellitus (15) & $35-50$ & $40.6 \pm 3.92$ & $<0.001$ \\
\hline & Smokers (15) & $28-43$ & $35.89 \pm 4.59$ & $<0.001$ \\
\hline & Obesity (15) & $34-46$ & $40.2 \pm 3.61$ & $<0.001$ \\
\hline & $\begin{array}{l}\text { Control (20) } \\
\text { (Reference group) }\end{array}$ & $46-64$ & $55.8 \pm 5.55$ & \\
\hline
\end{tabular}

ANOVA F: 59.917 df : $(5,94) \mathrm{P}<0.001$

As the $\mathrm{P}$ value is $<0.001$, the result is highly significant.

Table 14. Table Showing the levels of serum LDL in cases and controls.

\begin{tabular}{clcc}
\hline Vitamin & Group & Range of (mg\%) & Mean \pm S.D. (mg\%) \\
\hline & Myocardial Infarction (20) & $163-215$ & $193.5 \pm 14.56$ \\
& Hypertension (15) & $130-190$ & $165.8 \pm 19.71$ \\
& Diabetes Mellitus (15) & $155-184$ & $<0.001$ \\
Serum LDL & Smokers (15) & $126-187$ & $171.7 \pm 8.21$ \\
& Obesity (15) & $184-226$ & $160.7 \pm 18.68$ \\
& Control (20) & $119-139$ & $207.2 \pm 14.09$ \\
& (Reference group) & $<0.001$ & $128.9 \pm 6.36$ \\
\hline
\end{tabular}

ANOVA F: $66.931 \mathrm{df}:(5,94) \mathrm{P}<0.001$

As the $\mathrm{P}$ value is $<0.001$, the result is highly significant.

Table 15. Table Showing the levels of serum VLDL in cases and controls.

\begin{tabular}{|c|c|c|c|c|}
\hline Vitamin & Group & Range of (mg\%) & Mean \pm S.D. $(\mathrm{mg} \%)$ & P value \\
\hline \multirow{6}{*}{ Serum VLDL } & Myocardial Infarction (20) & $30-50$ & $39.8 \pm 5.21$ & $<0.001$ \\
\hline & Hypertension (15) & $21-46$ & $32.7 \pm 7.81$ & $>0.05$ \\
\hline & Diabetes Mellitus (15) & $21-52$ & $35.0 \pm 10.36$ & $>0.05$ \\
\hline & Smokers (15) & $35-49$ & $41.6 \pm 4.33$ & $<0.001$ \\
\hline & Obesity (15) & $34-67$ & $53.6 \pm 11.74$ & $<0.001$ \\
\hline & $\begin{array}{l}\text { Control (20) } \\
\text { (Reference group) }\end{array}$ & $25-34$ & $28.6 \pm 2.43$ & \\
\hline
\end{tabular}

ANOVA F: $22.535 \mathrm{df}:(5,94) \mathrm{P}<0.001$

Table 16. (A). Table Showing Total Cholesterol / HDL ratio in cases and controls.

\begin{tabular}{clcc}
\hline Vitamin & Group & Range & Mean \pm S.D. \\
\hline & Myocardial Infarction (20) & $4.9-7.6$ & $6.51 \pm 0.68$ \\
& Hypertension (15) & $4-6.9$ & $5.84 \pm 0.70$ \\
Total Cholesterol & Diabetes Mellitus (15) & $3.9-6.7$ & $<0.001$ \\
/ HDL & Smokers (15) & $5.1-7.9$ & $6.50 \pm 0.95$ \\
& Obesity (15) & $4.3-7.6$ & $6.27 \pm 0.92$ \\
& Control (20) & $2.6-3.9$ & $6.45 \pm 0.83$ \\
& (Reference group) & $<0.001$ & $3.17 \pm 0.37$ \\
\hline
\end{tabular}

ANOVA F: $54.312 \mathrm{df}:(5,94) \mathrm{P}<0.001$

As the $\mathrm{P}$ value is $<0.001$, the result is highly significant.

Table 16. (B). Table Showing LDL / HDL ratio in cases and controls.

\begin{tabular}{cllr}
\hline Vitamin & Group & Range & Mean \pm S.D. \\
\hline & Myocardial Infarction (20) & $4.9-6.3$ & $5.38 \pm 0.38$ \\
& Hypertension (15) & $3.3-4.9$ & $4.10 \pm 0.48$ \\
LDL / HDL & Diabetes Mellitus (15) & $3.1-4.8$ & $<0.001$ \\
ratio & Smokers (15) & $3.6-6.2$ & $4.24 \pm 0.39$ \\
& Obesity (15) & $4.1-5.9$ & $4.60 \pm 0.74$ \\
& Control (20) & $1.9-2.7$ & $5.14 \pm 0.51$ \\
& (Reference group) & $<0.001$ & $2.28 \pm 0.19$ \\
\hline
\end{tabular}

ANOVA F: 106.342 df : $(5,94) \mathrm{P}<0.001$ 
Table 17. Table Showing the levels of Blood Glucose in cases and controls.

\begin{tabular}{|c|c|c|c|c|}
\hline Vitamin & Group & Range of (mg\%) & Mean \pm S.D. $(\mathrm{mg} \%)$ & P value \\
\hline \multirow{6}{*}{ Blood Glucose } & Myocardial Infarction (20) & $75-122$ & $104.1 \pm 10.76$ & $>0.05$ \\
\hline & Hypertension (15) & $85-120$ & $105.6 \pm 9.98$ & $>0.05$ \\
\hline & Diabetes Mellitus (15) & $134-286$ & $204.2 \pm 42.37$ & $<0.001$ \\
\hline & Smokers (15) & $84-123$ & $108.2 \pm 10.00$ & $>0.05$ \\
\hline & Obesity (15) & $99-121$ & $110.2 \pm 6.10$ & $>0.05$ \\
\hline & $\begin{array}{l}\text { Control (20) } \\
\text { (Reference group) }\end{array}$ & $88-110$ & $99.3 \pm 6.36$ & \\
\hline
\end{tabular}

ANOVA F: $75.687 \mathrm{df}:(5,94) \mathrm{P}<0.001$

Table 18. A). Table Showing correlation coefficient between serum Liproprotein (a) and MDA

\begin{tabular}{lcc}
\hline Group & 'r' value & 'P' value \\
\hline Myocardial Infarction (20) & -0.500 & $<0.05 *$ \\
Hypertension (15) & 0.576 & $<0.05 *$ \\
Diabetes Mellitus (15) & 0.097 & $>0.05$ \\
Smokers (15) & 0.574 & $<0.05 *$ \\
Obesity (15) & 0.116 & $>0.05$ \\
\hline
\end{tabular}

* Statistically significant

Table 18. B). Table Showing correlation coefficient between serum Liproprotein (a) and GSH

\begin{tabular}{lcc}
\hline Group & 'r' value & 'P' value \\
\hline Myocardial Infarction (20) & -0.123 & $>0.05$ \\
Hypertension (15) & -0.243 & $>0.05$ \\
Diabetes Mellitus (15) & 0.158 & $>0.05$ \\
Smokers (15) & -0.659 & $<0.01 * *$ \\
Obesity (15) & -0.874 & $<0.001 * *$ \\
\hline
\end{tabular}

** Statistically highly significant

Table 18. C). Table Showing correlation coefficient between serum Liproprotein (a) and Vitamin $E$

\begin{tabular}{lcc}
\hline Group & 'r' value & 'P' value \\
\hline Myocardial Infarction (20) & -0.413 & $>0.05$ \\
Hypertension (15) & -0.465 & $>0.05$ \\
Diabetes Mellitus (15) & -0.694 & $<0.01 * *$ \\
Smokers (15) & -0.660 & $<0.01 * *$ \\
Obesity (15) & -0.813 & $<0.01 * *$ \\
\hline
\end{tabular}

** Statistically highly significant

Table 18. D). Table Showing correlation coefficient between serum Liproprotein (a) and Vitamin $C$

\begin{tabular}{lcc}
\hline Group & 'r' value & 'P' value \\
\hline Myocardial Infarction (20) & -0.004 & $>0.05$ \\
Hypertension (15) & -0.606 & $>0.05 *$ \\
Diabetes Mellitus (15) & -0.748 & $<0.01 * *$ \\
Smokers (15) & -0.246 & $>0.05$ \\
Obesity (15) & -0.735 & $<0.01 * *$ \\
\hline
\end{tabular}

* Statistically significant

** Statistically highly significant
Table 18. E). Table Showing correlation coefficient between serum Liproprotein (a) and Vitamin A

\begin{tabular}{lcc}
\hline Group & 'r'value & 'P' value \\
\hline Myocardial Infarction (20) & -0.116 & $>0.05$ \\
Hypertension (15) & 0.12 & $>0.05$ \\
Diabetes Mellitus (15) & 0.512 & $>0.05$ \\
Smokers (15) & 0.132 & $>0.05$ \\
Obesity (15) & -0.255 & $>0.05$ \\
\hline
\end{tabular}

As the $\mathrm{P}$ value is $>0.05$, the correlation is statistically not significant.

Table 18. F). Table Showing correlation coefficient between serum Liproprotein (a) and TC/HDL ratio

\begin{tabular}{lcc}
\hline Group & 'r' value & 'P' value \\
\hline Myocardial Infarction (20) & -0.342 & $>0.05$ \\
Hypertension (15) & 0.009 & $>0.05$ \\
Diabetes Mellitus (15) & 0.018 & $>0.05$ \\
Smokers (15) & -0.498 & $>0.05$ \\
Obesity (15) & 0.021 & $>0.05$ \\
\hline
\end{tabular}

As the $\mathrm{P}$ value $>0.05$, the correlation is statistically not significant.

The analysis of study group revealed that the Coronary heart diseases cases were between 30 to 60 years (Table-2). In Myocardial infarction cases, 51 to 60 years group maximum with $55 \%$ followed by 41 to 50 years group with $35 \%$. Age matched controls, 51 to 60 years group maximum with $45 \%$ followed by 41 to 50 years group with $30 \%$. It has been long known that ageing has a steady and consistent with atherosclerotic lesion. It is universally accepted that men are more prone to coronary atherosclerosis than women of child bearing age .After menopause, however women have equal risk of developing CHD.

Majority of the cases were from upper class with $50 \%$ followed by middle class with $30 \%$ (Table-3). As observed by many workers, the incidence of CHD is higher in person with high Socio-Economic status, while it is lower in low socio-economic groups. Majority of patient of myocardial infarction, $90 \%$ presented with chest pain followed by, $80 \%$ with sweating (Table-4), 45\% with vomiting, 30\% with breathlessness, $40 \%$ with palpitation and $20 \%$ with giddiness.

Results of routine investigation shows an increase in serum cholesterol, serum LDL, TC/HDL and LDL/HDL 
ratio were seen in all five groups including $\mathrm{MI}, \mathrm{HT}, \mathrm{DM}$, Smokers and Obesity cases, which were statistically significant. Serum Triglycerides were increased in MI, DM, Smokers and Obesity cases, which were statistically significant, where as significant increase was not seen in HT cases. Serum VLDL showed a statistically significant increase in MI, Smokers and Obesity cases, where as significant increase was not seen in HT and DM cases. Blood glucose showed a significant increase in DM cases, where as no significant increase were seen in MI, HT, Smokers and Obesity cases. A statistically significant decrease in serum HDL were seen in all five groups including MI, HT, DM, Smokers and Obesity cases. (Table $11,12,13,14,15,16$ (A) \& (B), 17)

In special investigations MDA and Lipoprotien (a) levels are increased in all five group including MI, HT, DM, Smokers and Obesity in comparison with levels in normal healthy controls (Table- $5 \&$ 6). The similar results were obtained by Boston.A.G.et al 1996, Sarah.H.W.et al 1996, Assman.G.et al 1998, Leo.J.S.et al 1999, Matthias.N. et al 2000, Rajeshekhar.D.et al 2004, who demonstrated a rise in serum Lipoprotein(a) levels in cases of CHD as compared to controls and who reported that elevated $\mathrm{Lp}(\mathrm{a})$ is an independent risk factor for the development of CHD.

Vitamin $\mathrm{E}$ and Vitamin $\mathrm{C}$ levels were decreased in all five groups including MI, HT, DM, Smokers and Obesity in comparison with levels in normal healthy controls.

Glutathione and Vitamin A levels were decreased in four groups including MI, HT, DM and Smokers in comparison with levels in normal healthy controls, where as in Obesity group the levels were not significant (Table-7).

Serum MDA levels were found to be higher in cases as compared to controls. This could be due to increased oxidative stress. We have also found a positive correlation between severity of disease and MDA levels.

Blood Glutathione, Serum Vitamin E, plasma Vitamin C and plasma Vitamin A levels were studied in patients of $\mathrm{CHD}$ and in patients with risk factor for CHD, as a measure of antioxidant status. Results showed a highly significant decrease in Glutathione, $\alpha$ - tocopherol, ascorbic acid and Vitamin A levels in cases as compared to controls. This could be indicative of increased need and a defective antioxidant mechanism in order to overcome the oxidative stress (Table- $8 \&$ 9).

An increased tendency to peroxidation of polyunsaturated fatty acids resulting from a reduction in anti oxidant availability might favour thrombosis.

In Hypertension group, serum MDA and serum Lipoprotein (a) levels are elevated and Blood Glutathione, serum Vitamin E, plasma Vitamin C and plasma Vitamin A levels were diminished, as compared to controls.

In smokers group, serum MDA and Serum Lipoprotein (a) values were increased and Blood Glutathione, serum Vitamin E, plasma Vitamin C and plasma Vitamin A values were diminished, as compared to controls.

In obesity group, serum MDA and Serum Lipoprotein (a) levels were elevated and serum Vitamin E and plasma Vitamin C levels were diminished, where as Blood Glutathione and plasma Vitamin A levels were not significant as compared to controls.

Thus, there were alterations in MDA, Lipoprotein (a) and anti-oxidant levels in cases of Coronary heart diseases and also in cases with risk factor for Coronary heart disease including hypertension, diabetes mellitus, smokers and obesity (Table 18, A, B,C,D,E \& F).

From the results, it can be conclude that serum MDA can be used to detect severity of Coronary heart diseases, as we have observed a significant decrease in blood glutathione, Vitamin E, Vitamin A with the increase in severity of oxidative stress.

A highly statistically significant increase in the serum Lipoprotein (a) was seen in cases as compared to controls. So we can conclude that Lipoprotein (a) is an independent risk factor for Coronary heart diseases.

It is therefore, concluded that MDA, Lipoprotein (a) and anti-oxidants like Glutathione, Vitamin E, Vitamin $\mathrm{C}$ and Vitamin A, be evaluated as bio-chemical parameters for preclinical assessment of "at risk group" for Coronary heart diseases and for assessing and monitoring cases of Coronary heart diseases.

Further studies with anti-oxidant Vitamin supplementation have to be performed to test the nature of association between high MDA, high Lipoprotein (a) and low anti-oxidants in Coronary heart diseases. And also further studies are required to evaluate the significance of serum Lipoprotein (a) estimation in the assessment of CHD risk of human subjects.

\section{References}

[1] Assmann G.,Cullen P.,Schulte H.,The Miinister Heart Study(PROCAM):results of fallow-up at 8 years.Eur Heart Journal 1998; 19( Suppl A): A2-A11.

[2] Boston A G.,Cupples L A.,Jenner J L.,Ordovas J M.,Seman L J.,Wilson P W.,Schaefer E J.,Castelli W P., Elevated plasma Lipoprotein (a) and Coronary heart disease in men aged 55 years and younger.A prospective study.JAMA 1996;276:544-548.

[3] Harrison's principle of Internal medicine,15th ed,2003.

[4] Leo J S.,Carl D.,Jennifer L J.,Adriene C L.,Judith R M.,Peter W F M.,William P C.,Jose M O.,Ernst J S., Lipoprotein(a)-cholesterol and coronary heart disease in the Framingham heart study. Clin Chem 1999;45:1039-1046.

[5] Matthias N.,Winfried M.,Heinrich W.,Is Lipoprotein(a) Cholesterol a significant indicator of cardiovascular risk?.Clin Chem 2000;46:436-437.

[6] Mc Cord J M.,Oxygen derived free radicals in postischaemic tissue injury.N Eng J Med 1985;312:159.

[7] P.S.M. 17th ed.Park JE, Park K,2003.

[8] Practical clinical Biochemistry,5th ed,Varley 1980. 
[9] Rajasekhar D.,Saibaba KSS.,Srinivasa Rao PVLN., Latheef SAA.,Subramanyam G., Lipoprotein(a): Better assessor of Coronary heart disease risk in South Indian population.Ind J Clin Biochem2004;19(2):53-59.

[10] Rath M.,Niendorf A.,Reblin T.,Dietel M.,Krebber HJ.,Beisiegel U.,Detection and quantification of Lipoprotein(a) in the arterial wall of 107 coronary bypass patients.Athersclerosis 1989;9:579-92.
[11] Sarah H W.,Stehen P F.,Santica M M.,A Prospective case control study of Lipoprotein(a) levels and Apo(a) size and risk of coronary heart disease in Stanford five-city project participants.Arterioscler Thromb Vasc Biol 1997;17:239245. 